\title{
Um estudo da relação entre o índice de acesso digital (DAI) e as vendas de uma empresa de compra de coletiva via internet no estado de São Paulo*
}

\author{
A study of the relation between the digital \\ access index (DAI) and the sales of aninternet \\ group-buying company from São Paulo state
}

Roberto Fava Scare ${ }^{1}$ Ildeberto Aparecido Rodello ${ }^{2}$ Leonardo Silva Antolini ${ }^{3}$ Flávio Notomi Kanazawa ${ }^{4}$

Recebido em: 14/07/2014

Aprovado em: 27/10/2014

1 Especialista em Estratégia de Marketing e Análise do Comportamento de Compra de Produtores Rurais. Professor de Marketing e Estratégia da FEA-RP/USP. Doutor e Mestre em Administração pela FEA/USP. Formado em Marketing pela ESPM e em Direito pelo Largo São Francisco. Membro da American Marketing Association (AMA) e da International Food and Agribusiness Management Association (IFAMA).

2 Professor do Depto. de Administração da FEA-RP/USP. Possui graduação em Tecnologia em Processamento de Dados pela Unicamp (1994), mestrado em Ciência da Computação pela UFSCar (1998) e doutorado em Física Aplicada (Computacional) pela USP (2003). Tem experiência na área de Tecnologia e Sistemas de Informação, com ênfase em sistemas ERP, sistemas de Realidade Virtual e Aumentada e Comércio Eletrônico.

Mestrando em Administração em Organizações pela FEA-RP/USP, campus Ribeirão Preto (2016). Possui graduação em Administração de Empresas pela FEA-RP/USP (2012), É Membro do Núcleo de Pesquisa em Agronegócios da FEA-RP (AgroFEA Ribeirão Preto). Estuda o Comportamento de Compra de Produtores Rurais, Canais de Distribuição e Acesso ao Mercado, Programas de Relacionamento e Planejamento e Gestão Estratégica de Marketing.

4 Mestrando em Administração em Organizações pela FEA-RP/USP, campus Ribeirão Preto (2016). Possui graduação em Administração de Empresas pela FEA-RP/USP (2012). Estuda Marketing Digital, Marca-país e Promoção de Destinos Turísticos.

\section{Resumo}

Recentemente, observou-se o surgimento e o desenvolvimento de organizações que popularizaram as compras coletivas via internet, ao operacionalizarem um modelo de negócios baseado no conceito de que uma elevação em escala da demanda pode resultar uma considerável queda nos preços para o consumidor. Por competir na Internet, a estratégia de comunicação de Marketing dessas empresas está essencialmente ligada a ela. Com relação às vendas, nota-se que todas as empresas têm somente um canal: a Internet. Assim, supõe-se que o consumidor deve ter acesso à Internet e que, quanto maior esse nível de acesso digital, maior será o potencial de vendas de um mercado. Considerou-se o Índice de Acesso Digital (DAI) como elemento de influência na construção efetiva do canal de vendas e utilizou-se da metodologia da International Telecommunication Union (ITU, 2003) para medir diversos fatores de acesso digital das cidades selecionadas no estudo. Nesse contexto, esta pesquisa propôs-se a estudar de forma aprofundada a relação entre as vendas de uma empresa de compra coletiva via internet e o DAI de cada mercado, especificamente, de três cidades do Estado de São Paulo, a fim de compreender mais a influência e os impactos do DAI nas vendas da empresa estudada. Identificou-se tendência positiva de relacionamento entre o DAI e as vendas da empresa analisada. Por fim, recomenda-se a utilização do DAI de forma estratégica, sendo uma variável que deve ser monitorada no planejamento de Marketing dessas empresas.

Palavras-chave: Compras coletivas. Índice de Acesso Digital. Comunicação de marketing. Promoção de vendas.

\begin{abstract}
Recently, we observe the founding and development of organizations that popularized the group-buying. They operationalize a business model based on the concept that an increase in scale of demand may result in a considerable drop in consumer prices. By competing on the Internet, the Marketing communication strategy of these companies is essentially linked to it. Regarding to sales, we note that all companies have only one sales channel: the Internet. Thus, it is assumed that the shopper should have Internet access and that the higher the level of digital access, the greater the sales potential of a market. We considered the Digital Access Index (DAI) as an element of influence in building effective sales channel and used the methodology of the International Telecommunication Union (ITU, 2003), to measure various factors of the digital access of selected cities in the study. Considering that, the study aimed to investigate in depth the relationship between sales of a group buying company and DAI of each market, specifically, three cities of São Paulo state, in order to understand more the influence and impacts of DAI on sales of these companies. Positive tendency was identified between the DAI and analyzed company's sales. Finally, we recommend the use of DAI in a strategic way, being a variable that must be monitored in the planning of Marketing of the group-buying companies.
\end{abstract}

Keywords: Group Buying. Digital Access Index. Marketing Communication. Sales Promotion. 


\section{Introdução}

$\mathrm{O}$ atual ambiente de negócios observa o surgimento e o desenvolvimento de organizações que popularizaram as compras coletivas via Internet ao operacionalizarem um modelo de negócios visando ao aumento da demanda baseado no conceito de ofertas de valor com preços atrativos, na maior disponibilidade de produtos e na de redução dos ciclos de baixa demanda decorrentes da sazonalidade de serviços. Hoje, o modelo de negócios de compra coletiva via Internet replicou-se por todo o mundo e o sucesso dessas organizações tornou-se evidente.

Esse modelo de negócios apoia-se nas mudanças que a Internet sofreu nos últimos anos em que as pessoas podem explorar, participar, construir ou afastar-se de qualquer comunidade online e também publicar conteúdo sem a necessidade de extenso conhecimento técnico (WIGAND; BENJAMIN; BIRKLAND, 2008). Dougherty, Musser e O'Reilly (2006) cunharam o termo Web 2.0 para definir esse novo paradigma e o descreveram como "um conjunto de tendências econômicas, sociais e tecnológicas que formam coletivamente a base da próxima geração da Internet - um meio distinto e mais maduro, caracterizado pela participação do usuário, abertura e efeitos de rede" (DOUGHERTY; MUSSER; O'REILLY, 2006. p. 4). Assim, segundo Rocha (2009), apesar do termo Web 2.0 ter conotações de uma nova versão da Internet, este não se refere a grandes atualizações em especificações técnicas ou estruturais, mas sim em uma mudança no modo como a Internet é encarada por usuários e desenvolvedores, tendo essa tendência impacto direto no desenvolvimento das empresas de compras coletivas via Internet.

Nesse sentido, a Web 2.0 proporciona um cenário com novas oportunidades de interação, produção de conteúdo e, consequentemente, novos modelos de negócio, graças ao potencial de criação e captura de valor dos agentes da rede, o que cria novas formas de consumo. Uma delas é o social shopping, que, segundo Stephen e Toubia (2010), é uma forma de mídia social baseada na Internet que permite a participação ativa de pessoas na comunicação e venda de produtos e serviços online.

Segundo Yin (2010), as compras coletivas integram essa nova modalidade de consumo na Internet. Segundo Santos e Cypriano, as empresas desse setor exploram o ambiente de negócios da Web 2.0, operando da seguinte maneira:

Uma vez definido um número mínimo de compradores para um determinado produto ou serviço e estipulado um prazo para que seja alcançado esse mínimo, o site publica ofertas que cobrem descontos de até $90 \%$. A validação da oferta fica condicionada ao alcance do número estipulado decompradores antes que o prazo termine. Em contagem regressiva, é dada a largada para que os interessados na oferta se empenhem em cooptar o máximo de indivíduos possível. Para tanto, os usuários da Internet recorrem à agilidade com que as redes sociais como Twitter, Orkut e Facebook fazem circular informações para divulgar aquela que parece ser uma oferta imperdível. A eficácia do procedimento se torna indissociável das dinâmicas que transcorrem em meio a essas redes e o número de adesões à compra coletiva vem atingindo proporções surpreendentes (SANTOS; CYPRIANO, 2010, p. 5).

Sob a ótica de abrangência geográfica de mercado, observa-se que as empresas de compras coletivas via internet atuam em três níveis de amplitude: internacional, nacional e regional. Os players globais, como o Groupon (http://www.groupon.com), e os nacionais, como o Peixe Urbano (http://www.peixeurbano.com.br), disponibilizam ofertas que podem ser consumidas em diversas regiões de um país, graças às alianças com fornecedores de produtos e serviços que lhes dão amplo acesso a redes de distribuição. Já as empresas com atuação regional oferecem produtos e serviços em um número menor de cidades, as quais a empresa de compra coletiva tem acesso à rede de distribuição dos fornecedores que atuam focados especificamente em determinadas regiões. Nota-se que as empresas com atuação nacional e internacional também disponibilizam ofertas no nível regional, tendo ofertas direcionadas e específicas para uma ou outra cidade. É, por essa razão, que o modelo de negócios das empresas de compras coletivas está intrinsecamente ligado à construção de alianças e redes de fornecedores que possam disponibilizar a oferta para os clientes de um mercado.

Lançar um novo produto em um mercado competitivo é uma tarefa complexa e desafiadora para o tomador de decisão, na qual tempo, informações incompletas, contexto organizacional e automotivação podem influenciar a decisão fortemente. Consequentemente, decisões erradas podem ser tomadas (STERMAN, 2000).

Dentre as diversas variáveis que os gestores das empresas de compras coletivas pela Internet devem monitorar ao decidir se entram em um ou outro mercado, está o nível de acesso à Internet e/ou outras tecnologias de informação e comunicação (TIC) que os consumidores de um mercado possuem. Para as empresas em questão, 
quanto maior esse acesso, maior será o potencial de negócios deste canal em um determinado mercado. Porém, ao invés de considerar o acesso à Internet como único elemento de influência na construção efetiva do canal de vendas das empresas de compra coletiva via Internet em determinado mercado alvo, faz-se necessária a seleção de fatores de acesso digital no intuito de estimar a sua relação com as vendas dessas empresas, pois monitorar somente o volume de acesso à Internet de um mercado pode ser pouco assertivo para essas empresas, uma vez que potencial o consumidor deve ter conhecimento suficiente para operar e absorver o seu conteúdo. Entende-se que diversos fatores se interrelacionam, influenciando o canal de vendas das empresas de compra coletiva via Internet, o que justifica a adoção de um índice para medi-los adequadamente.

A consolidação dos fatores traduz-se no Índice de Acesso Digital (ITU, 2003), que é calculado pela International Telecommunication Union e mede a capacidade geral dos indivíduos de um país, estado ou município em acessar e utilizar tecnologias de informação e comunicação. Esse índice mede 5 fatores considerados principais no acesso as TICs, a saber: infraestrutura, acesso, conhecimento, qualidade e utilização.

Esse estudo propõe-se a estudar a relação entre as vendas dos sites de compra coletiva e o índice de acesso digital (DAI) de cada mercado, especificamente, de cidades do Estado de São Paulo, no sentido de compreender a influência e os impactos desse indicador no potencial de negócios e de expansão para novos mercados das empresas estudadas. Nesse contexto, existe relação do DAI com as vendas das empresas de compra coletiva, e ainda, como o DAI pode ser utilizado estrategicamente por essas empresas?

\subsection{Objetivos}

O objetivo desse estudo é identificar a relação entre as vendas realizadas por meio de uma empresa de compras coletivas via internet e o DAI de municípios do Estado de São Paulo.

Busca-se ainda sugerir estratégias que relacionem o índice ao contexto de expansão do potencial negócio. Para isso, foram definidos três objetivos específicos:

- Obter as informações das vendas realizadas pela empresa de compra coletiva via internet da região selecionada;

- Calcular o DAI, de acordo com a metodologia oficial da ITU (2003), dos mercados em que a empresa atua, e;

- Verificar relação entre o potencial de negócios da empresa na respectiva região e o DAI estudada e como esse indicador pode ser utilizado por empresas de compra coletiva via internet.

\section{Referencial teórico}

Nessa seção discute-se o modelo de negócio que as empresas de compras coletivas na Internet utilizam, estudos já realizados sobre essas empresas e também estudos relacionados DAI.

\subsection{O modelo de negócios das compras coletivas na Internet}

As empresas de compra coletiva, ou compras em grupo, caracterizam-se por oferecerem produtos e serviços a preços significativamente reduzidos com a condição de que um número mínimo de compradores realize a compra.

Suportado pela lógica de que um maior número de compradores potencializa um maior número de ativação de ofertas, com auxílio das redes sociais, um comprador pode cooptar o máximo de indivíduos possíveis a fim de ativar a oferta, e assim, consumi-la. Não surpreendentemente, as ofertas se espalham de forma viral por meio de atualizações de redes sociais como o Twitter e Facebook,dentre outras,em uma base diária, incentivando pessoas a adquirirem a oferta. (DHOLAKIA, 2010).

A eficácia do procedimento se torna indissociável das dinâmicas que transcorrem em meio a essas redes e o número de adesões à compra coletiva vem atingindo proporções surpreendentes (SANTOS; CYPRIANO, 2010).

O modelo de negócios de compra coletiva pode ser enquadrado no modelo de plataforma multilateral, descrito por Einsenmann, Parker e Van Alstyne (2006). Segundo os autores, as empresas do setor bancário, software e mídia geram receita, construindo uma ligação entre diferentes agentes da sua rede. Os autores também citam que as redes construídas, com base em plataformas multilaterais, tendem a carecer de um lado que forneça o subsídio para outros agentes. É difícil para os fornecedores das plataformas multilaterais recuperarem os subsídios oferecidos ao cliente, fazendo com que vários tipos de plataformas dividam a taxa dos vendors na metade, 
uma para cada agente, como é o caso dos corretores de imóveis nos Estados Unidos.

De acordo com Benazzi e Pedra (2011), as empresas anunciantes geralmente recebem $50 \%$ do valor dos cupons vendidos, o restante é destinado aos gerenciadores dos sites. Os anunciantes não recebem os 50\% logo após a oferta, uma parcela é recebida em 30 dias e a outra após 60 dias, levando-se em consideração o uso efetivo dos cupons, ou seja, a receita daqueles que não foram utilizados fica com os administradores dos sites.

A plataforma de negócio das empresas de compra coletiva conecta empresas do varejo e prestação de serviços de diversos ramos e que estão dispostas a diminuir sua margem em troca de rápida obtenção de faturamento, com os consumidores altamente sensíveis a preço e que estão em busca de promoções de preço. Além dessa conexão com o cliente, a plataforma multilateral das empresas de compras coletivas utiliza-se capacidade de distribuição dos seus fornecedores para atingir um ou outro mercado.

O sucesso desse modelo de negócio se replicou por diversas regiões do mundo. No Brasil, segundo o site Bolsa de Ofertas (http://www.bolsadeofertas.com.br/), até 30 de novembro de 2010, existiam 246 sites de compra coletiva. Em 8 de dezembro de 2010, o número já havia saltado para 405. Em 31 de janeiro de 2011, o número era de 1025 sites.

É importante observar que esse notável crescimento aconteceu durante 10 meses após ter sido inaugurado o primeiro site de compras coletivas brasileiro, o Peixe Urbano (http://www.peixeurbano.com.br). Além disso, deve-se observar o surgimento de agregadores de sites de compra coletiva, em que o consumidor pode comparar as ofertas publicadas em cada um deles (SANTOS; CYPRIANO, 2010).

Porém, notou-se tendência de consolidação e maior profissionalização entre os players do país e, atualmente, segundo o site Bolsa de Ofertas, o número de empresas com participação relevante no mercado é de aproximadamente 50 .

Com relação a sua classificação, a compra coletiva é considerada uma ramificação do comércio eletrônico, denominada social shopping, na qual os compradores são envolvidos numa experiência de compra que utiliza a TIC na tentativa de mimetizar as interações sociais encontradas, por exemplo, em lojas e shopping centers. Além da compra coletiva, também fazem parte do social shopping: comunidades de compra, motores e ambientes de reco- mendação de produtos, social shopping marketplaces (tem alguma semelhança com bazares e feiras) e mecanismos de compra compartilhada.

Nesse contexto, social shopping pode ser definido como um ambiente de confiança onde um grupo influencia ativamente na decisão de compra de bens e serviços por meio de feedbacks positivos e negativos, revisões, avaliações e depoimentos sobre experiências passadas e presentes (STEPHEN; TOUBIA, 2010).

Arabshashi (2010) afirma que o fator-chave para essas empresas é a sua base de dados, especificamente, sua lista de contato de e-mails. $\mathrm{O}$ autor também afirma que essa base pode ser geograficamente segmentada e abranger significativa cobertura geográfica sem aumentos dos custos marginais, independentemente da localização do cliente. O valor oferecido para as empresas anunciantes é o elevado fluxo de consumidores ao longo de um curto período de tempo, utilizando-se de um serviço promocional, discriminação de preços e de um serviço pré-pago. (ARABSHASHI, 2010).

Porém, o elo com o cliente por si só não é suficiente, pois é necessário analisar se o fornecedor irá atingir ou não de forma efetiva o mercado que a empresa de compras coletivas na Internet deseja alcançar. Além disso, apesar de Arabshashi (2010) afirmar que não há aumento dos custos marginais com abrangência de significativa cobertura geográfica, nota-se que, se houver entrada em um mercado que não consiga absorver a oferta de valor dessas empresas e a remuneração em relação ao investimento, o sucesso dessas organizações será colocado em cheque. É necessário o entendimento de quais variáveis monitorar para entrada em um mercado para as empresas de compras coletivas na Internet, dentre elas, o DAI.

\subsection{Compras coletivas na Internet: estudos relacio- nados}

Considerando-se a literatura científica relacionada ao tema, poucos estudos relevantes foram realizados, com resultados ainda incipientes. Nessa seção são apresentados alguns dos trabalhos encontrados e que se julga serem importantes ao contexto da pesquisa.

Dholakia (2010) realizou pesquisa junto a organizações que ofereceram descontos pelo Groupon, principal player do setor, no intuito de elucidar qual era a efetividade das promoções do Groupon para as empresas anunciantes. O autor chega a resultados mistos: alguns empresários ressaltaram retornos positivos das promoções, 
enquanto outros se arrependeram de terem utilizado esse tipo de promoção de vendas.

Edelman, Jafe e Kominers (2010) procuraram entender os cupons eletrônicos de desconto ao nível econômico. Os resultados da pesquisa indicam que os cupons de desconto são naturalmente bons para certos tipos de negócios, e ruim para outros e que essas observações teóricas podem ajudar a interpretar a gama de reações aos descontos dos sites de compra coletiva.

Byers, Mitzenmacher e Zervas (2011), por sua vez, consolidaram as informações de venda do Groupon de 20 cidades americanas, no intuito de criar um modelo quantitativo de previsão do comportamento de vendas dessas ofertas. Os autores abordaram ainda a difusão social e impactos da reputação das empresas anunciantes, utilizando-se das redes sociais e de sites de recomendações.

Erdogmus e Çiçek (2011) realizaram 20 entrevistas com consumidores turcos, no intuito de analisar a compra coletiva naquele país. Os resultados mostram alguns drivers de valor e de custo valorizados entre os consumidores, como o montante e a porcentagem de desconto oferecido, questões sobre novidades na experimentação de produtos, tipos de produtos e serviços adquiridos, bem como fatores de honestidade percebidos pelos consumidores.

No Brasil, Santos e Cypriano (2010) analisaram as características da Web 2.0 e sua influência sobre o consumidor, ao valor adicionado por ele e pelos efeitos da rede e a eficácia da conectividade. Os autores também discorrem sobre as novas configurações de consumo em rede e as externalidades, financeiras ou não, que o modelo de negócios das compras coletivas gera.

Percebeu-se que, no que diz respeito à revisão de literatura relacionada, não há, nas principais fontes de artigos, revistas e periódicos, trabalhos com proposta semelhante a esse, ou seja, identificar se há relação nas vendas realizadas por meio de um site de compras coletivas e o DAI em diversos municípios do Estado de São Paulo e ainda sugerir estratégias que relacionem o índice ao contexto de expansão do potencial do negócio.

\subsection{O Índice de Acesso Digital - DAI}

Existem diversas metodologias que podem medir acesso e inclusão digital, como o Mapa da Exclusão Digital (NERI, 2003), o Economist Intelligence Unit (EIU, 2007), o National Informatization Quotient, do governo chinês (JIN; CHENGYU, 2002), o Digital Opportunity Index (ITU,
2005), o Digital Access Index (ITU, 2003), entre outros.

A metodologia escolhida para esse trabalho é a do Digital Access Index (DAI), ou Índice de Acesso Digital. A escolha desse modelo se deu devido a fatores como: utilização de dados secundários disponíveis na Internet, simplicidade e clareza, credibilidade do órgão criador do índice e aceitação do modelo internacionalmente.

A fórmula do DAI (Equação 1) foi elaborada pela International Telecommunication Union (ITU) e mede a capacidade geral dos indivíduos de um país em acessar e utilizar tecnologias de informação e comunicação. Ela é composta por oito variáveis, agrupadas em cinco categorias. Além disso, há um valor ideal determinado pela ITU (2005) para cada variável que é usado como comparação no cálculo do índice de uma determinada região. O Quadro 1 apresenta a composição do Índice de Acesso Digital.

Quadro 1 - Composição do Índice de Acesso Digital (DAI)

\begin{tabular}{|c|c|c|c|}
\hline Categoria & Variável & $\begin{array}{l}\text { Ideal } \\
\text { ITU }\end{array}$ & Peso \\
\hline \multirow{2}{*}{ Infraestrutura } & $\begin{array}{l}\text { Número de assinantes de } \\
\text { telefones fixos a cada } 100 \\
\text { habitantes }\end{array}$ & 60 & 0,1 \\
\hline & $\begin{array}{l}\text { Telefones celulares a cada } 100 \\
\text { habitantes }\end{array}$ & 100 & 0,1 \\
\hline Acessibilidade & $\begin{array}{l}\text { Preço da internet como } \\
\text { porcentagem da renda per } \\
\text { capita }(1-\mathrm{x} \%)\end{array}$ & 100 & 0,2 \\
\hline \multirow[b]{2}{*}{ Conhecimento } & Taxa de Alfabetização & 100 & 0,13 \\
\hline & $\begin{array}{l}\text { Combinado das matrículas } \\
\text { escolares de } 1^{\circ}, 2^{\circ} \text { e } 3^{\circ} \text { grau. }\end{array}$ & 100 & 0,7 \\
\hline \multirow{2}{*}{ Qualidade } & $\begin{array}{l}\text { Internet internacional (Mbits } \\
\text { per capita) }\end{array}$ & 10.000 & 0,1 \\
\hline & $\begin{array}{l}\text { Assinantes de banda larga a } \\
\text { cada } 100 \text { habitantes }\end{array}$ & 30 & 0,1 \\
\hline Utilização & $\begin{array}{l}\text { Usuários de Internet a cada } \\
100 \text { habitantes }\end{array}$ & 85 & 0,2 \\
\hline DAÎ́ & \multicolumn{2}{|c|}{$\begin{array}{l}\text { Média simples dos resultados das } 5 \\
\text { categorias de variáveis }\end{array}$} & 1 \\
\hline
\end{tabular}

Fonte: ITU (2005)

A classificação final é feita em quatro níveis: alto (DAI $=0,7$ ou mais), superior (DAI $=0,5$ a 0,69 ), médio (DAI $=0,3$ a 0,49$)$ e baixo (DAI $=0,29$ ou menos). Ressalta-se que, oficialmente, a ITU (2005) somente divulga o DAI para países.

No Brasil, Bonilha (2006) realizou a estratificação do DAI para estados, partindo-se da mesma metodologia da ITU (2003). O autor identificou diferenças significativas dentre os diferentes estados brasileiros. Na revisão de literatura relacionada, não se encontrou estudos do DAI para municípios brasileiros, mas verificou-se que há disponibili- 
dade de informações em órgãos do governo brasileiro e, sendo assim, é possível calcular do DAI para o nível municipal.

\section{Método}

No estudo, foi realizada uma pesquisa descritiva, que, segundo Cooper e Schindler (2003), é uma "pesquisa com ênfase em solução prática de problemas, na qual um problema é uma circunstância negativa ou uma oportunidade". Observou-se a oportunidade de verificar a possível relação nas vendas realizadas por meio de um site de compra coletiva e o DAI. O método definido da amostragem foi o não probabilístico por conveniência. Na técnica de amostragem não probabilística, procura-se obter uma amostra dos elementos convenientes: o pesquisador é quem seleciona as unidades amostrais (MALHOTRA, 2001).

Sendo assim, tomou-se como objeto de estudo uma empresa que atua significativamente em três cidades do interior paulista, especificamente: Ribeirão Preto, Franca e Presidente Prudente. A empresa foi denominada "empresa A", por questões de confidencialidade. Segundo o site Deal Explorer (http://www.dealexplorer.com), a “empresa A" está entre as 10 maiores empresas em faturamento do setor e é um dos principais players na região onde atua, considerando o ano de 2012.

Foi utilizado o modelo não experimental que é quando como explicam Sampieri, Collado e Lucio (2006), as variáveis independentes não são propositalmente manipuladas. Quanto ao aspecto temporal, realizou-se uma pesquisa transversal, que estuda o fenômeno em um determinado momento, de acordo com Kerlinger (1980). Em relação à natureza, realizou-se uma pesquisa quantitativa, analisando-se dados coletados da empresa estudada e suas relações com o índice de acesso, calculado a partir de fontes secundárias.

As variáveis, suas siglas, fontes de dados e ano de publicação da informação para o cálculo do DAI no Brasil são apresentados no Quadro 1. Segundo a ITU (2003), a fórmula (Equação 1) para o cálculo do DAI é uma somatória das variáveis apresentadas no Quadro 1.

Equação 1 - Fórmula para o cálculo do DAI

$\mathrm{DAI}=\mathrm{TELFIX} 0,1+\mathrm{TELCEL}^{*} 0,1+\mathrm{PREÇO}^{*} 0,20+$ ALFAB $^{*} 0,13+$ MATRI $^{*} 0,7+$ BITS $^{*} 0,1+$ BANDA $^{*} 0,1+$ USUARIOS*0,2

Fonte: ITU (2003)
Quadro 2 - Variáveis e Fontes de Dados para o Cálculo do DAI

\begin{tabular}{|c|c|c|c|}
\hline Sigla & Variável & Fonte & Ano \\
\hline TELFIX & $\begin{array}{l}\text { Número de assinantes de te- } \\
\text { lefones fixos a cada } 100 \text { ha- } \\
\text { bitantes }\end{array}$ & Anatel & 2011 \\
\hline TELCEL & $\begin{array}{l}\text { Telefones celulares a cada } \\
100 \text { habitantes }\end{array}$ & Anatel & 2011 \\
\hline PREÇO & $\begin{array}{l}\text { Preço da internet como por- } \\
\text { centagem da renda per capita } \\
(1-\mathrm{x} \%)\end{array}$ & $\begin{array}{l}\text { Cetic.br } \\
\text { / IBGE }\end{array}$ & 2010 \\
\hline ALFAB & Taxa de Alfabetização & SEADE & 2000 \\
\hline MATRI & $\begin{array}{l}\text { Combinado das matrículas } \\
\text { escolares de } 1^{\circ}, 2^{\circ} \text { e } 3^{\circ} \text { grau. }\end{array}$ & $\begin{array}{l}\text { Inep / } \\
\text { IBGE }\end{array}$ & 2009 \\
\hline BITS & $\begin{array}{l}\text { Internet internacional (bits } \\
\text { per capita) }\end{array}$ & Anatel & 2011 \\
\hline BANDA & $\begin{array}{l}\text { Assinantes de banda larga a } \\
\text { cada } 100 \text { habitantes }\end{array}$ & Anatel & 2011 \\
\hline \multirow[t]{2}{*}{ USUARIOS } & $\begin{array}{l}\text { Usuários de internet a cada } \\
100 \text { habitantes }\end{array}$ & Anatel & 2011 \\
\hline & População & IBGE & 2010 \\
\hline
\end{tabular}

Fonte: Elaborado pelos autores

Quanto aos fins, foi uma pesquisa exploratória, que Gil (2002) fala que tem como objetivo:

[...] proporcionar maior familiaridade com o problema, com vistas a torná-lo mais explícito ou a constituir hipóteses. Pode-se dizer que estas pesquisas têm como objetivo principal o aprimoramento de ideias ou a descoberta de intuições. Seu planejamento é, portanto, bastante flexível, de modo que possibilite a consideração dos mais variados aspectos relativos ao fato estudado (GIL, 2002, p. 41).

Em relação aos meios, esse trabalho utilizou a pesquisa bibliográfica e coleta e análise de dados secundários. A primeira, ainda segundo Gil (2002), é desenvolvida com base em material já elaborado, constituído principalmente por livros e artigos científicos de qualidade e mais largamente utilizada em pesquisas exploratórias. Grande cuidado e atenção foram tomados ao decorrer do estudo quanto à seleção das fontes da literatura abordada neste estudo e utilizada para compor o referencial teórico.

\section{Resultados}

Nessa seção são apresentados os resultados obtidos pela pesquisa realizada. É feita uma breve caracterização da Empresa. Os dados obtidos são seguidos da sua análise e consequente discussão. 


\subsection{Caracterização da empresa}

A empresa estudada tem sede na cidade de Ribeirão Preto e atua significativamente em três cidades do interior de São Paulo, especificamente: Ribeirão Preto, Franca e Presidente Prudente. Fundada em 2010, a empresa foi pioneira na atuação na compra coletiva na região de Ribeirão Preto. Segundo dados do site Deal Explorer (http://www.dealexplorer.com) de 2012, a "empresa A" está entre as 10 maiores empresas em faturamento do setor e é um dos principais players na região que atua. Ela também está constantemente no ranking Top 50 do site Bolsa de Ofertas. A participação no estudo deu-se em troca da confidencialidade do nome empresa.

\subsection{Apresentação dos dados obtidos}

Inicialmente, realizou-se a coleta e a consolidação das vendas da "empresa A" para as cidades de Franca, Ribeirão Preto e Presidente Prudente. A evolução das vendas da "empresa A" nas respectivas cidades durante o ano de 2011 é exibida no Gráfico 1.

Gráfico 1 - Faturamento por Cidade 2011 - Empresa A

Faturamento por cidade 2011- Empresa A

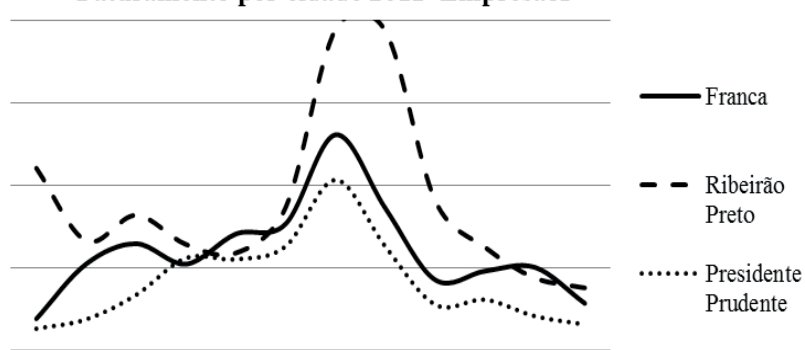

Fonte: Elaborado pelos Autores com dados fornecidos pela "empresa A"

É possível observar picos de faturamento, como no mês de julho, por exemplo, e baixas, como no mês de setembro. É importante ressaltar que não foi objetivo da pesquisa identificar os motivos que ocasionaram isso. Assim, calculou-se a venda média mensal de cada cidade para o ano de 2011 e, após esse dado, foi dividido pelo número de habitantes de cada cidade, com base nos dados do Censo Demográfico do Instituto Brasileiro de Geografia e Estatística (IBGE, 2011). Calculou-se o montante vendido per capita, como forma de identificar o nível de vendas médio mensal por habitante de cada cidade, como mostra a Tabela 1.
Tabela 1 - Venda per capita - média mensal - 2011 - Empresa A

\begin{tabular}{|l|c|r|r|}
\hline Cidade & $\begin{array}{c}\text { Venda Média } \\
\text { Mensalem } \\
\mathbf{2 0 1 1}\end{array}$ & População & $\begin{array}{c}\text { VendaMédia per } \\
\text { capita 2011(R\$ per } \\
\text { capita) }\end{array}$ \\
\hline Franca & $\mathrm{R} \$ 60.992,28$ & 321.012 & 0,15 \\
\hline $\begin{array}{l}\text { Ribeirão } \\
\text { Preto }\end{array}$ & $\mathrm{R} \$ 91.850,50$ & 612.339 & 0,19 \\
\hline $\begin{array}{l}\text { Presidente } \\
\text { Prudente }\end{array}$ & $\mathrm{R} \$ 41.425,00$ & 207.625 & 0,20 \\
\hline
\end{tabular}

Fonte: Elaborado pelos autores com base no IBGE (2011) e em dados fornecidos pela "empresa A"

A média permite identificar que a cidade de Presidente Prudente tem uma população menor que a de Franca, mas que o faturamento per capita é maior. O DAI de cada cidade foi então calculado no intuito de realizar a comparação entre esse índice e as vendas per capita da "empresa A". Nota-se que as três cidades apresentam DAI de classificação superior, ou seja, de 0,5 a 0,69, conforme indica a ITU. A Tabela 2 exibe o Índice de Acesso Digital calculado para as cidades analisadas.

Tabela 2 - Índice de Acesso Digital (DAI) das Cidades Analisadas

\begin{tabular}{|lcl|}
\hline Cidade & $\begin{array}{c}\text { DAI - Índice de } \\
\text { Inclusão Digital }\end{array}$ & Classificação \\
\hline Franca & 0,61 & Superior \\
\hline Ribeirão Preto & 0,66 & Superior \\
\hline Presidente Prudente & 0,65 & Superior \\
\hline
\end{tabular}

Fonte: Elaborado pelos autores

No intuito de realizar um cruzamento inicial do DAI e do indicador de venda per capita, construiu-se um gráfico de dispersão com os índices de venda per capita e de acesso digital (Gráfico 1). Dessa forma, tem-se a relação inicial entre as vendas per capita das cidades estudadas e seus respectivos DAI. É possível observar uma tendência de relacionamento que sugere ser positiva entre as variáveis. A cidade de Presidente Prudente tem um DAI calculado em 0,65 e faturamento per capita de $\mathrm{R} \$ 0,20$, enquanto Franca, que tem o menor DAI $(0,61)$, tem menor faturamento per capita. Dessa maneira, nas cidades analisadas, é possível relacionar que quanto maior o DAI, maiores podem ser as vendas per capita. 
Gráfico 2 - DAI x Venda Média Mensal per capita - Empresa A - 2011

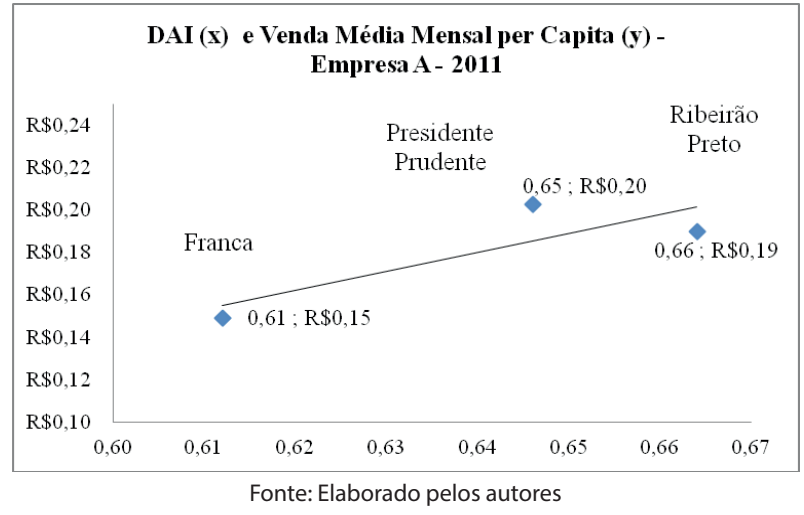

\subsection{Discussão dos resultados}

Foram obtidas informações sobre as vendas realizadas por uma empresa de compra coletiva via internet da região de Ribeirão Preto, escolhidas cidades de atuação da empresa e calculado o índice de acesso digital para cada uma delas, de forma a realizar o cruzamento dessas informações, como traçado nos objetivos do trabalho.

Assim, identificou-se que há uma tendência positiva na relação entre o DAI e as vendas da empresa, sugerindo que nas cidades onde o DAI é superior, a tendência é que a venda mensal per capita seja maior. Portanto, é interessante notar que, estrategicamente, seja interessante para uma determinada empresa de compra coletiva via internet ter conhecimento do DAI da cidade onde esteja com intenção de ampliar seus negócios ou mesmo investir em estratégia de divulgação e comunicação.

Ressalta-se que esse trabalho tem limitações por ser introdutório e ter um número de cidades e empresas estudadas bastante limitado, mas que pode contribuir para a linha de pesquisa da área de gestão de compra coletiva via internet.

\section{Conclusões}

Como mencionado, o objetivo deste estudo foi identificar a relação entre as vendas realizadas por meio de uma empresa de compras coletivas via internet e o DAI de municípios atuantes por essa empresa, do Estado de São Paulo. Identificou-se possível tendência de relacionamento entre o DAI e as vendas da empresa analisada. Porém, não se pode afirmar que o resultado apresenta correlação estatisticamente significativa, devi- do ao limitado número de cidades pesquisadas. Embora a "empresa A" atue em mais 8 cidades, além das que foram analisadas nesse estudo, as vendas nessas cidades não se mostraram significativas, o que não enriqueceria as análises.

Destaca-se que o presente trabalho é um estudo descritivo que visa elucidar as relações iniciais do tema abordado e que a literatura científica relacionada ao tema ainda constrói seus passos iniciais. O cálculo do DAI para o nível municipal também é inédito na literatura científica brasileira e, ao longo do estudo, percebeu-se que esse índice pode ser útil para diversas áreas de pesquisa.

Com relação a sugestões e recomendações para as empresas de compra coletiva via internet, ressalta-se que o DAI pode ser uma das variáveis a ser considerada no estabelecimento da estratégia de Marketing, contribuindo para a formação efetiva do canal de vendas, e consequentemente, impactando positivamente sobre os resultados das empresas. Por exemplo, se uma empresa deseja entrar em um novo mercado e estimar quais serão os potenciais resultados de venda, deve considerar diversos fatores: um deles pode ser o DAI.

Outro ponto que pode ser abordado é a utilização do DAI para definição do potencial de mercado de uma região, por exemplo: se as vendas per capita da cidade $\mathrm{X}$ são menores do que as de $\mathrm{Y}$ e o DAI de $\mathrm{X}$ maior do que $\mathrm{Y}$, a cidade $\mathrm{X}$ pode estar sendo subexplorada pela empresa, uma vez que se identificou tendência de relação positiva entre o DAI e a compra coletiva.

Por fim, recomenda-se a utilização do DAI de forma estratégica pelas empresas em questão, combinado com as variáveis que já fazem parte do planejamento estratégico de Marketing dessas empresas.

\section{Referências}

AMERICAN MARKETING ASSOCIATION. Index. Disponível em: <http://www.marketingpower.com>. Acesso em: set. 2011.

AGÊNCIA NACIONAL DE TELECOMUNICAÇÕES. Index. Disponível em: <http://www.anatel.gov.br>. Acesso em: jan. 2013.

ARABSHASHI, A. Undressing Groupon: an analysis of the Groupon business model. 2010. Disponível em: <http:// www.ahmadalia.com/download/Undressing-Groupon. pdf>.Acesso em: set. 2011. 
BELCH, G. E. ; BELCH, M.A. Propaganda e promoção. 7. ed. São Paulo: McGraw-Hill, 2008.

BONILHA, C. DAI -Digital Access Index: Brazil breakdown. ITU, 2006. Disponível em: <http://www.itu.int/ ITU-D/ict/dai/material/BR_DAI_ITU.pdf $>$. Acesso em: jul. 2010.

BYERS, J. W; MITZENMACHER, M; ZERVAS, G. Daily Deals: prediction, social diffusion, and reputational ramifications. 2011. Cornell University Library. Disponível em: <http://arxiv.org/abs/1109.1530>. Acesso em: jul. 2010.

CENTRO DE ESTUDOS SOBRE AS TECNOLOGIAS DA INFORMAÇÃO E DA COMUNICAÇÃO. Index. Disponível em: <http://cetic.br/>. Acesso em: jan. 2013.

COOPER, D. R.; SCHINDLER, P. S. Métodos de pesquisa em administração. 7. ed. São Paulo: Bookman, 2003.

DHOLAKIA, U. M. How effective are Groupon promotions for business? Rice University, 2010. Disponível em: $<$ http://www.ruf.rice.edu/ dholakia $>$. Acesso em: set. 2011.

DOUGHERTY, D.; MUSSER, J.; O'REILLY, T. Web 2.0: principles and best practices. Sebastopol: O'Reilly Radar, 2006.

EDELMAN, B; JAFFE, J; KOMINERS, S. C. To Groupon or not to Groupon: the profitability of deep discounts. Harvard Business School, 2010. Disponível em: <http://www. scottkom.com/research.html>. Acesso em: set. 2011.

EINSENMANN, T; PARKER, G; VAN ALSTYNE. Strategies for Two-Sided markets. Harvard Business Review On Point. oct , 2006.

ECONOMIST INTELLIGENCE UNIT. Index. Disponível em: <http://www.eiu.com>. Acesso em: jan. 2012.

ERDOGMUS, I. E; ÇIÇEK, M. Online Group buying: what is there for the consumers? INTERNATIONAL STRATEGIC MANAGEMENT CONFERENCE. 7., 2011, Paris. Anais... Paris, 2011.

GIL, A. C. Como elaborar projetos de pesquisa. 4. ed. São Paulo: Atlas, 2002.

INSTITUTO BRASILEIRO DE GEOGRAFIA E ESTATÍSTICA. Index. Disponível em: <http://www.ibge.gov. br>. Acesso em: jan. 2013.

INSTITUTO NACIONAL DE ESTUDOS E PESQUISAS EDUCACIONAIS ANÍSIO TEIXEIRA. Index. Disponível em: <http://www.inep.gov.br>. Acesso em: jan. 2013.
INTERNATIONAL TELECOMMUNICATION UNION. Digital Access Index, 2003. Disponível em: <http://www. itu.int/ITU-D/ict/dai/>. Acesso em: jan. 2013.

JIN, J; CHENGYU, X. The digital divide in terms of national informatization quocient (NIQ): the perspective of mainland China. In: INTERNATIONAL CONFERENCE ON DIGITAL DIVIDES: TECHNOLOGY AND POLITICS IN THE INFORMATION AGE, 2002, Hong Kong: Anais... Hong Kong: Baptist University, 2002.

KERLINGER, F. N. Metodologia da pesquisa em ciências sociais: um tratamento conceitual. São Paulo: EPU/ EDUSP, 1980.

KOTLER, P; KELLER, K. L. Administração de marketing. 12. ed. São Paulo: Pearson Prentice Hall, 2006.

MALHOTRA, N. K. Pesquisa de marketing. Porto Alegre: Bookman, 2001.

MCCARTHTY, E. J. Basic marketing: a managerial approach. Homewood, Ill: IRWIN, R. D, 1960.

NERI, M. C. Mapa da exclusão digital. Rio de Janeiro: FGV/IBRE, CPS, 2003.

PANCRAZIO, P. S. Promoção de vendas: o gatilho do marketing. São Paulo: Futura, 2010.

PINHO, J. B. Comunicação em marketing: princípios da comunicação mercadológica. Campinas: Papirus, 2001.

ROCHA, C. T. C. Mídia digital internet e a democratização da tecnociência: novo paradigma do acesso aberto. Revista de Ciência da Informação, Rio de Janeiro, v. 10, n. 3, jun. 2009.

SAMPIERI, R. H.; COLLADO, C; F.; LUCIO, P; B. Metodologia de pesquisa. 3. ed. São Paulo: Mc Graw-Hill, 2006.

SANTOS, F. C.; CYPRIANO, C. P. Novas configurações de consumo em rede: dissipação e criação de valor. ENCONTRO NACIONAL DE ESTUDOS DO CONSUMO. 5,. 2010, Rio de Janeiro. Anais... Rio de Janeiro, 2010.

SEADE. Fundação Sistema Estadual de Análise de Dados. Disponível em: <http://www.seade.gov.br >. Acesso em: jan. 2013.

SHIMP, T. A. Comunicação integrada de marketing: propaganda e promoção. Porto Alegre: Bookman, 2009.

STEPHEN, A, T.; TOUBIA, O. Deriving value from social commerce networks. Journal of Marketing Research, Chicago, v. 17. p. 215-228, 2010.

STERMAN, J. D. Business dynamics: systems thinking 
and modeling for a complex world. Boston: Irwin/Mcgraw-Hill, 2000.

STRAUSS, J.; FROST, R. E-Marketing. 6. ed. São Paulo: Pearson Prentice Hall, 2012.

VAZ, C. A. Google marketing: o guia definitivo de marketing digital. São Paulo: Novatec, 2010.
WIGAND, R. T.; BENJAMIN, R. I.; BIRKLAND, J. L. Web 2.0 and beyond: implications for electronic commerce. New York: ICEC '08 Proceedings of the $10^{\text {th }}$ international conference on Electronic Commerce, 2008.

YIN, E. Social shop till you drop: a quick primer. Gigaom. Disponível em <http:/gigaom.com/2010/07/11/social-commerce/>.Acesso em: jul. 2011. 